\title{
Influence of spatial variability on 3D slope failures
}

\author{
M. A. Hicks, J. Chen \& W. A. Spencer \\ School of Mechanical, Aerospace and Civil Engineering, \\ The University of Manchester, UK
}

\begin{abstract}
This paper considers the influence of spatial variability of undrained shear strength $\left(c_{u}\right)$ on the stability of long slopes cut in clay. Random fields of $c_{u}$ are mapped onto finite element meshes used in Monte Carlo analyses, and slope performance is quantified in two ways: (a) reliability is computed as a function of the global factor of safety, $F$ (based on the mean property value); and (b) volumes of material associated with potential slides are discussed with respect to the probability of failure. By plotting contours of displacement and shear strain invariant at failure, slide volumes are shown to be influenced by the depthdependency of the statistics of $c_{u}$ in 2D analysis and, additionally, by the type of failure mode in 3D analysis. Spatial variability can occasionally result in slope failure at relatively high values of $F$, although the associated risk may then be relatively low due to the greater likelihood of more localised failure.
\end{abstract}

Keywords: finite elements, reliability, risk, slope stability, spatial variability, stochastic analysis.

\section{Introduction}

Spatial variability of material properties affects soil behaviour and geo-structural performance [1]. It also causes uncertainty about actual ground conditions, and leads to the need for probabilistic analysis and measures of response [2]. These include reliability, which is the probability of failure not occurring, and risk, which is the probability of failure $\times$ consequence of failure. This paper considers reliability and risk for the problem of 3D slope stability. In particular, it uses finite elements and stochastic analysis to investigate the influence, on slope performance, of the spatial variability of undrained shear strength $\left(c_{u}\right)$. 


\subsection{Background}

The spatial variability of $c_{u}$ may be represented by its statistics. These are the mean $(\mu)$ and standard deviation $(\sigma)$, which define the coefficient of variation, $V$ $=\sigma / \mu$, and the scale of fluctuation $(\theta)$, which is a measure of the distance over which property values are strongly correlated. The horizontal scale of fluctuation $\left(\theta_{h}\right)$ is generally larger than the vertical scale of fluctuation $\left(\theta_{v}\right)$, and the degree of anisotropy of the variability is $\xi=\theta_{h} / \theta_{v}$.

Paice and Griffiths [3] and Griffiths and Fenton [4,5] used finite elements and stochastic analysis to investigate $2 \mathrm{D}$ slope reliability. They assumed a depthindependent, lognormal distribution for $c_{u}$, and used local average subdivision [6] to generate isotropic $(\xi=1)$ random fields of $c_{u}$. Hicks and Samy [7-10] performed similar 2D analyses, but used a normal distribution for $c_{u}$, arguing that, for practical ranges of $V(0.1-0.3)$, the chances of negative property values were negligible. They considered both anisotropic $(\xi>1)$ and depth-dependent random fields of $c_{u}$, and described a method for deriving reliability-based (problem-dependent) characteristic values in line with EC7 [9-10].

Spencer and Hicks [11], Spencer [12] and Hicks and Spencer [13] used parallel computing [14] to investigate the reliability of 3D slopes. This paper reviews their principal findings. It then considers the risk posed by $3 \mathrm{D}$ slope failures, by investigating the link between probability of failure and potential slide volumes.

\section{3D modelling of slope reliability}

Figure 1 shows details of the $45^{\circ}, 3 \mathrm{D}$ slope analysed by Spencer and Hicks [11]. It is $H=5 \mathrm{~m}$ high, $L=100 \mathrm{~m}$ long and rests on a firm base, and is modelled using 20-node brick finite elements with $2 \times 2 \times 2$ Gaussian integration [15]. The boundary conditions comprise a fixed base and rollers on the back face preventing displacement in the $x$ direction. The two sides have rollers allowing only vertical $(z)$ displacement, as allowing displacement in the $x$ direction was found to cause a bias in slope failures near the mesh ends. Hicks and Spencer [13] demonstrated the validity of the boundary conditions by investigating slopes of different length.
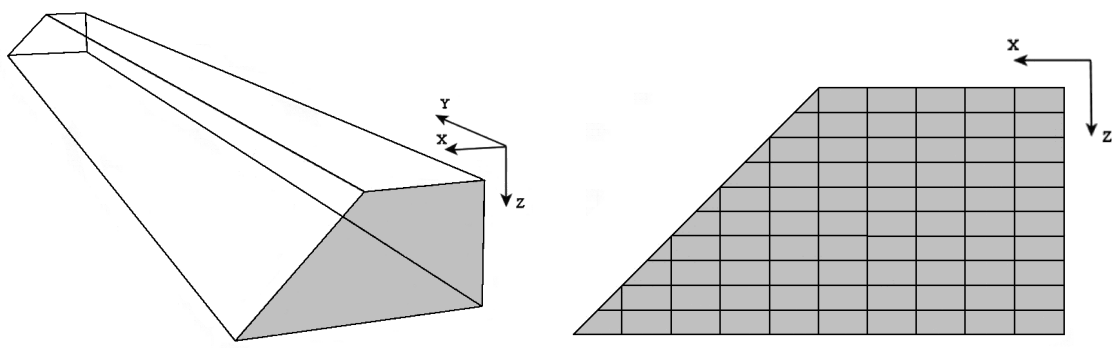

Figure 1: Problem geometry and cross-section mesh details. 
The soil is modelled as an elastic, perfectly plastic Tresca material, and is characterised by a spatially varying undrained shear strength defined at the Gauss point level. Random fields of $c_{u}$ are generated using local average subdivision [6,7], and by assuming a normal distribution, a depth-independent mean $(\mu)$ and coefficient of variation $(V=0.3)$, and vertical and horizontal scales of fluctuation $\left(\theta_{v}=1.0 \mathrm{~m}\right.$ and $\left.\theta_{h}\right)$.

The method of analysis is described by Spencer and Hicks [11] and is similar to previous 2D studies (but with some changes to improve efficiency). In brief, slope reliability $R$ is computed as a function of the global factor of safety $F$ (i.e. based on $\mu$ ) by Monte Carlo simulation. For a given $V, \theta_{v}$ and $\theta_{h}, N$ random fields of $c_{u}$ are generated. The problem domain is then analysed for each field in turn, by applying gravity loading to generate the in situ stresses. This is done for progressively lower values of $\mu$ (and hence $F$ ) until the slope fails.

For a given value of $F, R=\left(1-N_{f} / N\right) \times 100$, in which $N_{f}$ is the number of realisations in which slope failure occurs at a global factor of safety equal to, or greater than, $F$. Figure 2 summarises the influence of $\xi\left(=\theta_{h} / \theta_{v}\right)$ on the results, for both (a) 2D and (b) 3D idealisations of the problem. For 2D modelling (using $N=1000$ ), there is a wide distribution of $F$ for all values of $\xi$, due to the plane strain condition implying an infinite scale of fluctuation in the third dimension. The solution is also highly dependent on the degree of anisotropy of the heterogeneity, although it converges at higher values of $\xi$ [7-10]. In contrast, for 3D modelling (using $N=500$ ) there is no such convergence. Moreover, Spencer and Hicks [11] identified three possible failure modes. These are illustrated in Figure 3, which shows typical deformed meshes and contours of horizontal (outof-face) displacement at failure. They are summarised as follows:

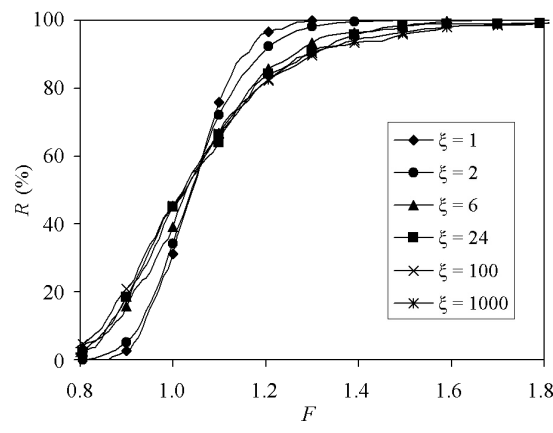

(a) 2D Analyses

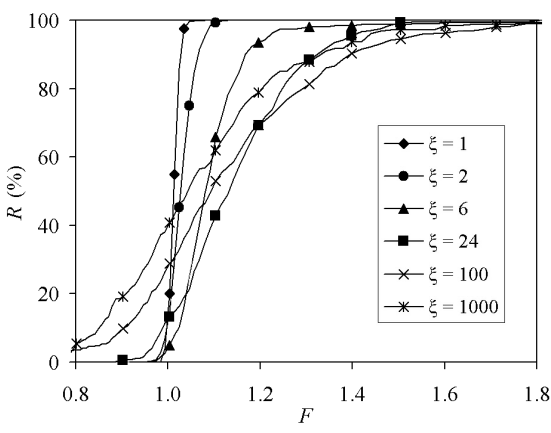

(b) 3D Analyses

Figure 2: $\quad$ Reliability versus global factor of safety [11].

(a) Mode 1: For $\theta_{h}<H$, there are no semi-continuous weaker zones along which failure can propagate. Hence, failure mechanisms pass through strong and weak zones alike, the average $c_{u}$ on the rupture surface approaches $\mu$, and the range of solutions (across the realisations) is narrow. The solution is similar to a $2 \mathrm{D}$ deterministic solution based on $\mu$; i.e. the failure mechanism initiates 
from the toe and extends along the length of the slope, and $R$ increases rapidly from $0-100 \%$ as $F$ passes through 1.0 .

(b) Mode 2: For $H<\theta_{h}<L / 2$, discrete failures are likely as failure is attracted to semi-continuous weaker zones. As $L$ increases (relative to $\theta_{h}$ ) $R$ decreases, due to the increased likelihood of encountering a zone weak enough to trigger failure. Hicks and Spencer [13] showed that simple probability theory can be used to extrapolate the results of detailed stochastic analyses (such as in this paper) to predict the performance of slopes of different length.

(c) Mode 3: For $\theta_{h}>L / 2$, the soil takes on a layered appearance and failure extends along the length of the slope, rather like Mode 1. However, in contrast to Mode 1 there is a wide range of solutions, as the location of the slide is influenced by the locations of the weaker layers. This case is analogous to a $2 \mathrm{D}$ stochastic analysis, as is seen by comparing the relevant curves in Figures 2(a) and 2(b).

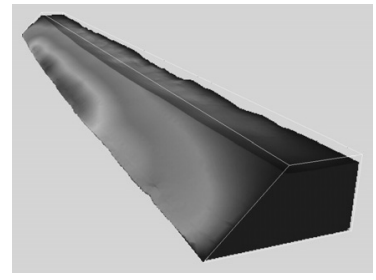

(a) Mode 1

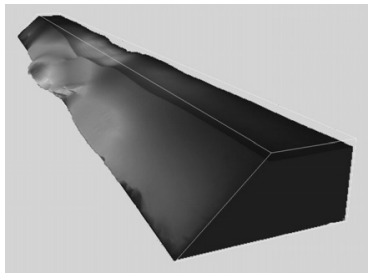

(b) Mode 2

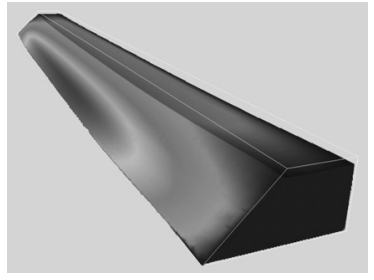

(c) Mode 3

Figure 3: $\quad$ Typical failure mechanisms [11].

\section{Modelling of risk}

The Spencer and Hicks [11] analyses have been revisited to investigate the risk associated with potential slides. If risk is the probability of failure $\times$ consequence of failure, it can be quantified by equating consequence with slide volume.

\subsection{D analysis}

For 2D analyses, the slide volume has been estimated from contours of shear strain invariant at failure. An imaginary point in space, well above the centre of the slope face, was chosen. Next, a series of lines radiating out from this point (across the slope mesh) was considered. The maximum value of shear strain invariant along each line was detected and, by connecting the locus of maxima from all the lines, the rupture surface was defined. Figure 4 shows the contours of shear strain invariant for one realisation and the back-figured rupture surface. The slide volume is simply the volume of material above the rupture surface.

Figure 5(a) shows the distribution of slide volumes, as a percentage of the mesh volume, for $\xi=6$ (for $N=400$ ). The relatively narrow range of solutions is due to the importance of slope height on slope stability when $\mu$ is constant with depth and $V$ is not large (although note that some of the larger volumes are due 
to interaction between the mechanism and vertical boundary). That is, failure will tend to initiate from or near the slope toe, as highlighted by the distribution of "slide depths" in Figure 5(b). In contrast, Figure 6 shows distributions for a slope in which $\mu$ is directly proportional to depth (with $V$ constant). For this special case, slope stability is independent of slope height and failure can initiate from any point along the slope surface [7], as is evident from the approximately uniform distribution in Figure 6(b). For the two examples illustrated in Figures 4-6, there was no obvious link between probability of failure and slide volume; i.e. the distribution of possible volumes was similar for all failure probabilities.
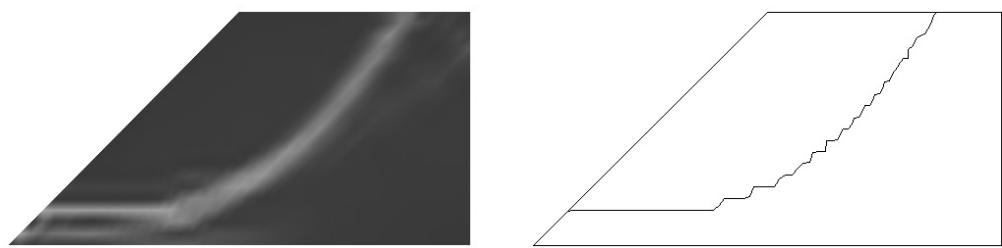

Figure 4: $\quad$ Typical failure mechanism for constant $\mu$.

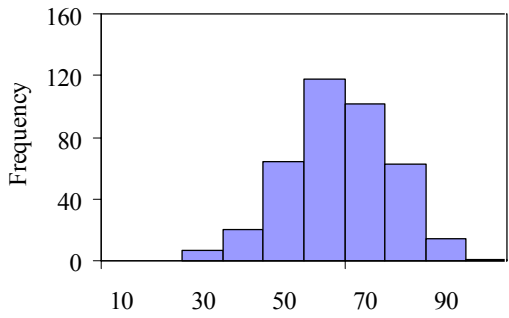

(a) Slide Volume (\%)

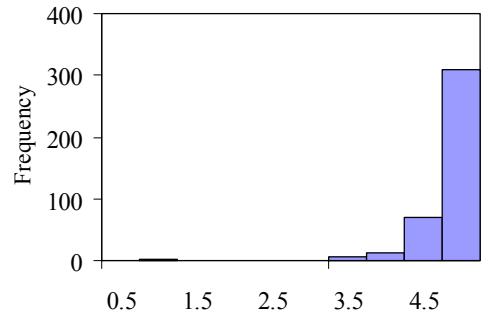

(b) Slide Depth (m)

Figure 5: $\quad$ Frequency distributions for constant $\mu$.

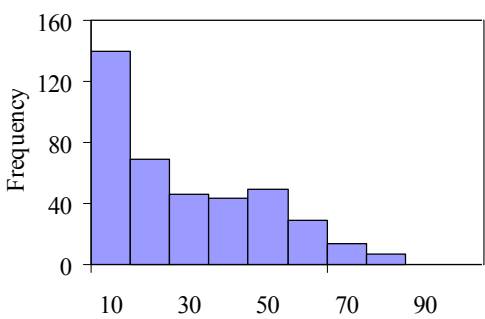

(a) Slide Volume (\%)

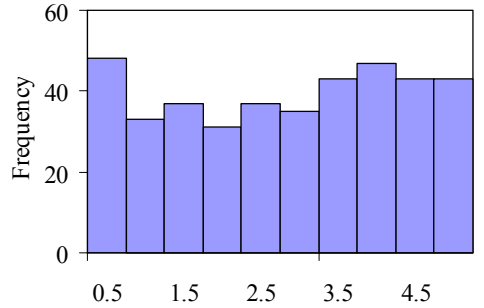

(b) Slide Depth (m)

Figure 6: $\quad$ Frequency distributions for $\mu \propto$ depth. 


\subsection{D analysis}

The estimation of slide volumes is harder in 3D, especially for Mode 2 type failures. It is still possible to compute contours of shear strain invariant, but three factors make the automated computation of slide volumes more difficult: (a) the heterogeneity of $c_{u}$ can trigger the initiation of multiple failure mechanisms at the local scale, which sometimes makes the global failure mechanism harder to identify; (b) the development of more than one slide along the length of the slope; and (c) the interaction of two or more slides.

A simple method has been adopted to enable a crude comparison of slide volumes. For each realisation the maximum nodal displacement at slope failure was recorded. The slide volume was then taken to be the volume of all elements exhibiting a displacement greater than $15 \%$ of the maximum nodal displacement. This approximate percentage was based on the 2D deterministic solution (based on $\mu$ ); i.e. by finding out what percentage of the maximum displacement gave a volume comparable with that found by computing the rupture surface using the shear strain invariant contours. The approach has been validated by comparing the $2 \mathrm{D}$ deterministic mechanism with the "slide volume" predicted for $3 \mathrm{D}$ using the $15 \%$ threshold.

Figure 7 shows two curves for reliability versus factor of safety for $\xi=12$; the original curve from Spencer [12] and a new curve derived by re-analysing the problem for a further 500 realisations. Also shown are the estimated slide volumes for the latest set of analyses, expressed as a percentage of the total mesh volume. Although the figure demonstrates the wide range of slide volumes possible for different probabilities of failure, it is also apparent that, for higher $F$, the volumes are much smaller.

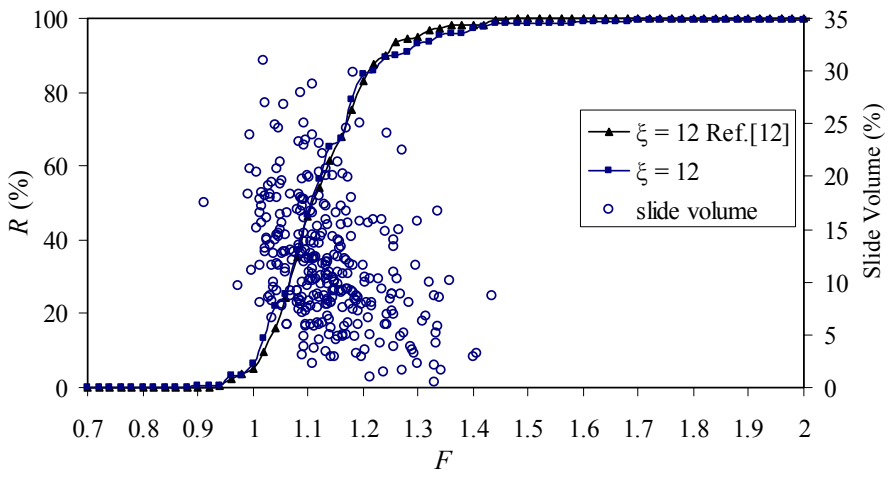

Figure 7: Reliability and slide volume versus global factor of safety.

The wide range of possible mechanisms is illustrated in Figure 8, which shows displaced meshes and contours of horizontal (out-of-face) displacement for nine realisations. The estimated slide volume percentages seem reasonable for the individual mechanisms. (Note that the slide volume for Mode 1 was about 50\%.) 


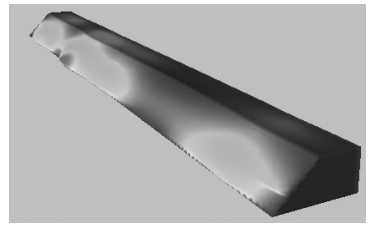

(a) $18 \%$

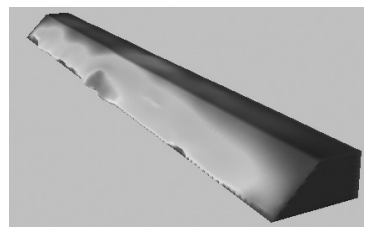

(d) $25 \%$

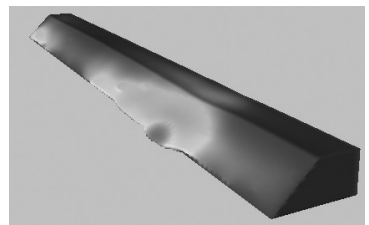

(g) $9 \%$

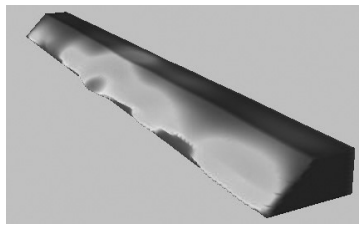

(b) $27 \%$

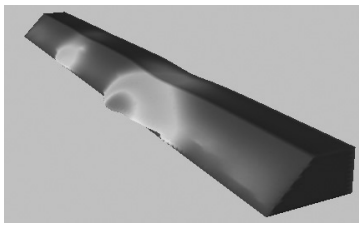

(e) $6 \%$

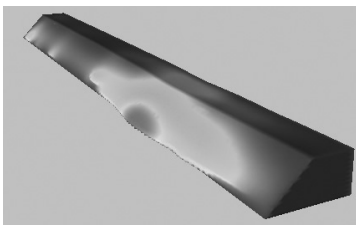

(h) $16 \%$

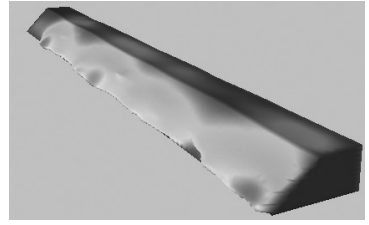

(c) $31 \%$

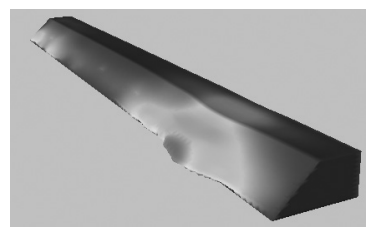

(f) $6 \%$

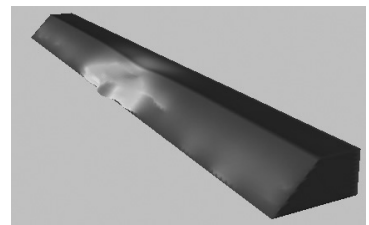

(i) $1 \%$

Figure 8: $\quad$ Example Mode 2 failure mechanisms and estimated slide volumes.

\section{Conclusions}

The paper has highlighted the need for 3D analysis of spatially variable slopes. 2D analysis implies an infinite scale of fluctuation in the third dimension and considers only one failure mode. In contrast, 3D analysis leads to three possible failure modes, which depend on the value of $\theta_{h}$ relative to slope geometry. Modes 1 and 3 are extreme modes, analogous to 2D deterministic and stochastic solutions, respectively. Mode 2 includes single, multiple and interacting discrete failures, and leads to a level of reliability that is function of slope length. For this case, there is wide range of possible slide volumes for most probabilities of failure. However, the risk associated with higher global factors of safety may be relatively low, due to the low probability of failure and a decreased likelihood of large slide volumes.

\section{Acknowledgement}

This work has been funded by EPSRC (Grant Reference, EP/D037247/1). 


\section{References}

[1] Hicks, M. A. \& Onisiphorou, C., Stochastic evaluation of static liquefaction in a predominantly dilative sand fill. Géotechnique, 55(2), pp. 123-133, 2005.

[2] Hicks, M. A., (editor). Risk and Variability in Geotechnical Engineering, Thomas Telford, ICE: London, 2007.

[3] Paice, G. M. \& Griffiths, D. V., Reliability of an undrained clay slope formed from spatially random soil. Proc. 9th Int. Conf. Computer Methods and Advances in Geomech., Wuhan, China, 1, pp. 543-548, 1997.

[4] Griffiths, D. V. \& Fenton, G. A., Influence of soil strength spatial variability on the stability of an undrained clay slope by finite elements. Slope Stability 2000, Proc. Sessions of Geo-Denver 2000, ASCE, pp. 184-193, 2000.

[5] Griffiths, D. V. \& Fenton, G. A., Probabilistic slope stability analysis by finite elements. ASCE J. Geotech. and Geoenviron. Eng., 130(5), pp. 507518, 2004.

[6] Fenton, G. A. \& Vanmarcke, E. H., Simulation of random fields via local average subdivision. ASCE J. Eng. Mech., 116(8), pp. 1733-1749, 1990.

[7] Hicks, M. A. \& Samy, K., Influence of heterogeneity on undrained clay slope stability. Quarterly J. Eng. Geology and Hydrogeology, 35(1), pp. 41-49, 2002.

[8] Hicks, M. A. \& Samy, K., Influence of anisotropic spatial variability on slope reliability. Proc. 8th Int. Symp. Num. Models in Geomech., Rome, Italy, pp. 535-539, 2002.

[9] Hicks, M. A. \& Samy, K., Reliability-based characteristic values: a stochastic approach to Eurocode 7. Ground Eng., 35(12), pp. 30-34, 2002.

[10] Hicks, M. A. \& Samy, K., Stochastic evaluation of heterogeneous slope stability. Italian Geotech. J., 38(2), pp. 54-66, 2004.

[11] Spencer, W. A. \& Hicks, M. A., A 3D finite element study of slope reliability. Proc. 10th Int. Symp. Num. Models in Geomech., Rhodes, Greece, pp. 539-543, 2007.

[12] Spencer, W. A., Parallel Stochastic and Finite Element Modelling of Clay Slope Stability in 3D, University of Manchester, UK, 2007.

[13] Hicks, M. A. \& Spencer, W. A., 3D finite element modelling of slope reliability. Proc. 5th ECCOMAS, Venice, Italy, (under review) 2008.

[14] Hicks, M. A., Nuttall, J. D. \& Spencer, W. A., Stochastic and finite element modelling of 3D heterogeneity in geo-engineering. Proc. ECCOMAS Thematic Conf. Heterogeneous Materials with Applications in Construction and Biomedical Engineering, Prague, Czech Republic, pp. 236-237, 2007.

[15] Smith, I. M. \& Griffiths, D. V., Programming the Finite Element Method, 4th edition, Wiley: Chichester, 2004. 